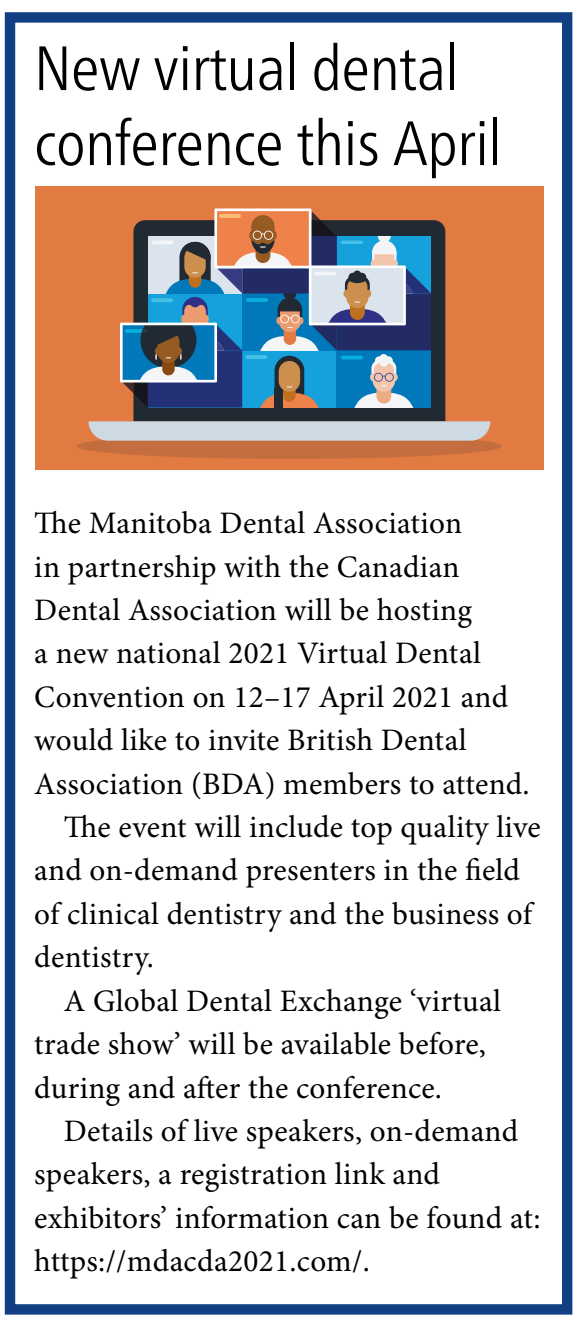

\title{
GDC introduces option to pay ARF by instalments
}

The General Dental Council has announced a new pay by instalments option for the annual retention fee (ARF), designed to support dental professionals who pay their own fee by enabling them to spread the cost over the year with quarterly direct debits. For dental care professionals (DCPs) this works out as four payments of $£ 28.50$ and for dentists it is four payments of $£ 170$.

To take advantage of the option in 2021, DCPs need to log in to eGDC from early May and select the quarterly direct debit option no later than 31 May 2021. The regulator is working to get this new option set up and says it will be in touch with DCPs when they are able to sign up.

GDC Chief Executive and Registrar, Ian Brack, said: 'We know that many dental professionals want more flexibility in how they pay the ARF. Detailed feasibility work has been completed and work is underway to make the necessary changes to our systems to allow us to offer this to DCPs this year. We expect this work to be complete by early May and DCPs who want to pay quarterly will then need to sign up by 31 May. Detailed guidance will follow nearer the time'.

The British Society of Dental Hygiene and Therapy (BSDHT) and the British Association of Dental Nurses (BADN) have welcomed the GDC's decision. BADN President Jacqui Elsden said: 'BADN have been lobbying the GDC for some years to allow payment by instalments, but the answer was always that this would be too costly, and additionally that it was not possible under the current legal framework'

BADN believe that $£ 114$ a year is still too high a fee for dental nurses.

Ms Elsden said: 'We hope that now the GDC have acknowledged the financial burden the ARF places on dental nurses, they will give further consideration to BADN's two other proposals: that the ARF be lowered to a more realistic figure for dental nurses, and that a lower ARF be implemented for all dental professionals, not just dental nurses, who work part time'.

Dentists who wish to pay their ARF by instalments will be able to select this option later in the year.

\section{New US toothpaste significantly improves gum health}

Scientists at the University of California Irvine's Beckman Laser Institute \& Medical Clinic have conducted the first study to show that brushing with a novel toothpaste improves symptoms in patients with periodontitis.

While several recent studies show that individuals with chronic gum disease are more likely to experience potentially life-threatening complications if they contract COVID-19, a new study shows promise in addressing the main issue. Researchers at the UCI Beckman Laser Institute \& Medical Clinic recently reported results of a six-month long study, showing for the first time that a novel toothpaste demonstrated medically significant improvements in the health of the gums of patients with periodontitis, the most severe form of gum disease. ${ }^{1}$

As a part of her focus on translational research, Dr Petra Wilder Smith initiated a double-blinded study testing a novel dental gel, LivFresh Dental Gel, against an FDA-approved anti-gingivitis toothpaste, to investigate their effects on gum health in patients with early to moderate periodontitis. The six-month long study compared how the two toothpastes affected periodontal pocket depths, gingival inflammation, and gum bleeding in patients with periodontitis who were in maintenance care.

The findings revealed that subjects who brushed with LivFresh Dental Gel experienced clinically and statistically significant improvements in their symptoms versus the control group that brushed with an over-thecounter, FDA-approved anti-plaque, antigingivitis toothpaste.

Several previous laboratory and clinical studies by Wilder-Smith's group have demonstrated that the novel formulation retards on a molecular level dental plaque formation, attachment and re-accumulation at the tooth surface by increasing its negative charge. The charged surface prevents early individual plaque islands from coalescing into larger deposits, discourages plaque from attaching to the tooth surface and supports the breakup of existing plaque deposits. Thus, by inhibiting dental plaque, the novel formulation reduces the presence of the harmful plaque bacteria and bacterial products that are implicated in chronic gum disease.

In individuals who brushed with the test gel, pocket depths in the gums improved in more than $80 \%$ of diseased sites. Additionally, subjects who brushed with the new formulation had 2.5 times less gum inflammation and 1.9 times less gum bleeding, when compared to the group using the conventional toothpaste.

Dr Wilder-Smith said: 'We anticipate that this novel formulation, when used in combination with professional periodontal care, may revolutionise healing in the gums and maintenance of periodontal health' Larger and longer studies are now in progress to solidify these findings.

\section{Reference}

1. Kaur M, Geurs N C, Cobb C M et al. Evaluating efficacy of a novel dentifrice in reducing probing depths in stage I and II periodontitis maintenance patients: A randomized, double-blind, positive controlled clinical trial. J Periodontol 2020; doi: 10.1002/JPER.20-0721. 